\title{
Current-induced cutting and recombination of magnetic superconducting vortex loops in mesoscopic superconductor-ferromagnet heterostructures
}

\author{
G. R. Berdiyorov, ${ }^{1}$ M. M. Doria, ${ }^{2}$ A. R. de C. Romaguera, ${ }^{3}$ M. V. Milošević, ${ }^{1}$ E. H. Brandt, ${ }^{4, *}$ and F. M. Peeters ${ }^{1, \dagger}$ \\ ${ }^{1}$ Departement Fysica, Universiteit Antwerpen, Groenenborgerlaan 171, B-2020 Antwerpen, Belgium \\ ${ }^{2}$ Universidade Federal do Rio de Janeiro, 68.528, Rio de Janeiro - RJ, 21941-972, Brazil \\ ${ }^{3}$ Departamento de Física, Universidade Federal Rural de Pernambuco, 52171-900 Recife, Pernambuco, Brazil \\ ${ }^{4}$ Max-Planck-Institut für Metallforschung, D-70506 Stuttgart, Germany
}

(Received 22 September 2012; revised manuscript received 29 April 2013; published 20 May 2013)

\begin{abstract}
Vortex loops are generated by the inhomogeneous stray field of a magnetic dipole on top of a current-carrying mesoscopic superconductor. Cutting and recombination processes unfold under the applied drive, resulting in periodic voltage oscillations across the sample. We show that a direct and detectable consequence of the cutting and recombination of these vortex loops in the present setup is the onset of vortices at surfaces where they were absent prior to the application of the external current. The nonlinear dynamics of vortex loops is studied within the time-dependent Ginzburg-Landau theory to describe the profound three-dimensional features of their time evolution.
\end{abstract}

DOI: 10.1103/PhysRevB.87.184508

PACS number(s): 74.78.Fk, 73.23.-b, 74.25.Wx, 74.78.Na

Introduction. One of the most interesting phenomena in liquid and gaseous matter is the development of vortices. Much effort has been put forward to understand their effect on the underlying physics ranging from the atmospheric to the nanoscale. A consequence of the filamentous nature of vortices is the existence of cutting and recombination. ${ }^{1}$ This may be induced by several reasons, such as vortex-vortex collision, the interaction of vortices with boundaries, or their crossing across a weak link. ${ }^{2-4}$ Cutting and recombination processes in classical fluids are described by the Navier-Stokes equations, ${ }^{5-7}$ but in superfluids such processes become more interesting, ${ }^{8-12}$ because of the quantization of the flux carried by the vortices. The conservation of circulation limits the phase space of quantized vortices, rendering their collision simpler than in a classical fluid. In type II superconductors vortex lines display a variety of features not found in neutral superfluids because of their charged nature. ${ }^{13}$

In this work we propose a method to detect cutting and recombination processes of vortex lines in type II superconductors. Our system consists of a thick superconducting film with an in-plane oriented ferromagnetic dot on top, separated by an insulating oxide layer from the film (see Fig. 1). The magnetic dot is the only source of magnetic field, and so, curved vortex lines arise and end at the top surface due to the large size of the sample. Initially there are no vortex lines reaching the bottom and the lateral sides of the superconductor, ${ }^{14}$ but the presence of a current applied perpendicularly to the magnetic moment plane changes this. Vortex loops evolve due to the Lorentz force, undergo cutting and recombination, and reach the surfaces. The evolving vortex lines can be stopped by additional pinning centers put strategically at the surfaces of the thick film to allow their direct static observation, as they were not found there before the application of the current drive.

The understanding of cutting and recombination processes is also important for different reasons ranging from basic physics to technological applications. ${ }^{15}$ It is well known that the motion of vortices is the main source of energy dissipation in current carrying superconductors. Therefore vortex motion must be avoided by all means to obtain an increase of the critical current of the material. ${ }^{16}$ Cutting and recombination processes play an unsuspected and important role in dissipation processes ${ }^{17}$ due to the entanglement of vortices inside bulk superconductors. For example, consider vortices in the presence of a homogeneous three-dimensional (3D) matrix of strong pointlike pinning centers. In this case a segment of a vortex line, although pinned in its extremities, undergoes distortion because of the external current and may eventually interact with a nearby similarly pinned segment of a distinct vortex line. Near to the external surfaces and edges of a superconductor cutting and recombination processes play an even more important role, as the entrance and exit of vortices can occur in the form of naturally curved lines that render the effects of an external applied current more dramatic. ${ }^{18}$

The present proposal for the detection of cutting and recombination processes of vortex lines is analyzed through the time-dependent Ginzburg-Landau (GL) theory where curved vortices naturally arise and evolve as extended 3D objects. Nevertheless our results can be qualitatively understood from the Lorentz force, which acts over all segments of a naturally curved vortex, $\int \mathbf{J} \times d \boldsymbol{\Phi}_{\mathbf{0}}$ with $\Phi_{0}=h c / 2 e$ the flux quantum, and is opposed by the line tension caused by the elastic properties of the line. Locally the external applied current $\mathbf{J}$ acts on the segment $d \boldsymbol{\Phi}_{\mathbf{0}}$ of the vortex line, which feels the local Lorentz force $\mathbf{J} \times d \boldsymbol{\Phi}_{\mathbf{0}}$ that competes with the line tension, causing global distortion of the line. ${ }^{16} \mathrm{By}$ its turn the motion of the segment with velocity $\mathbf{v}$ produces a local electrical field $\mathbf{E}$ as given by the Josephson relation $\mathbf{v} \times \mathbf{E}$. All these features are naturally incorporated into the GL approach, able to describe the time evolution of vortex lines in the presence of an external applied current. The expansion or the contraction of the vortex loop naturally follows from this scenario since it is a direct consequence of the sign and the strength of the applied current. The final outcome is the onset of vortices at the bottom and (or) the side surfaces of the sample, not previously found in the static limit. The resulting vortices produce measurable signals that allow the identification of the underlying cutting and recombination process. Thus in our setup the action of the 


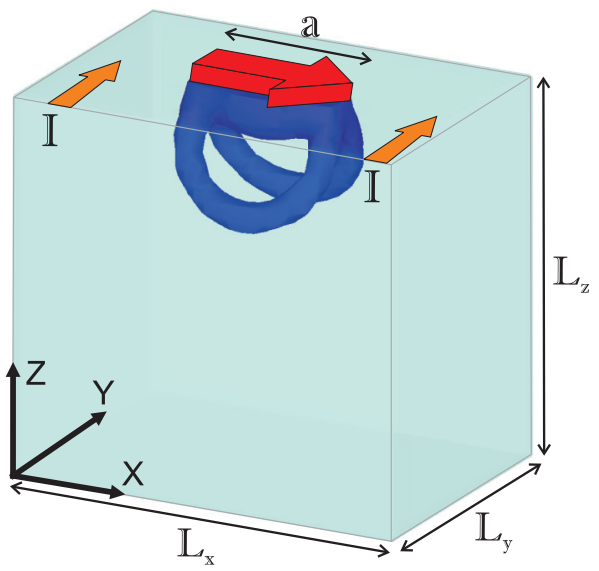

FIG. 1. (Color online) Model system: a superconducting sample (of size $L_{x} \times L_{y} \times L_{z}$ ) with an in-plain magnetic dipole (of length $a$ and magnetic moment $m$ ) on top. The external current (injected through the normal contacts in the $x-z$ plane at $y=0$ and $y=L_{y}$ ) flows in the $y$ direction $\left(j^{+}\right)$and $-y$ direction $\left(j^{-}\right)$.

Lorentz force on curved 3D vortex lines is a key ingredient to obtain vortex cutting and recombination processes not found in previous studies done in superconducting-magnet hybrid structures, ${ }^{19}$ which were limited to two-dimensional dynamics of vortices in thin films. The present study considers a reversible regime where vortex lines freely evolve in time under the Lorentz force. Pinning near to the bottom is also included in our numerical analysis.

The here proposed method for cutting and recombination processes is unique and different from all previous ones since it is not based on the crossing of the vortex line on a weak link, and solely relies on the effects of the Lorentz force on curved lines. The first studies of cutting and recombination processes were carried on a superconducting cylinder with current flowing along the central axis, in the same direction of the applied magnetic field. ${ }^{15,20}$ In this case a local misalignment between the current and a vortex line segment triggers further deformation of the vortex line leading to a longitudinal resistivity. This local deformation is a consequence of a weak link spot, according to many, ${ }^{21-24}$ and works as the kernel for the instability that leads to the growth of a vortex loop that eventually crosses the original vortex line. Cutting and recombination processes have also been studied through the Giaver experiment, ${ }^{2-4}$ which requires that the vortex line moves perpendicular to a three-layer system, where the intermediate layer is made of a distinct material which plays the role of a weak link. Recently Palau et al. ${ }^{25}$ extended this idea to allow the Lorentz force to exceed the line tension in the weak layer, such that deformations of the vortex lines can occur without limit, cross-joining itself to free an expanding loop. Vortex cutting and recombination processes though not directly observed there, provided the best qualitative explanation for their results. ${ }^{20,25}$

Theoretical approach. Our setup consists of an extreme type-II superconducting mesoscopic rectangular block (size $L_{x} \times L_{y} \times L_{z} \leqslant \lambda$ ) having an extended magnetic dipole (of length $a$, momentum $m$ on top), oriented along the surface ( $x$ direction), separated from the superconductor by an insulating layer of thickness $(\delta=1 \xi)$, and with transport current applied through the normal contacts (see Fig. 1). The dynamical properties of vortices follow by considering the time-dependent GL equation ${ }^{26}$ below:

$$
u\left(\frac{\partial}{\partial t}+i \varphi\right) \psi=(\nabla-i \mathbf{A})^{2} \psi+\left(1-|\psi|^{2}\right) \psi,
$$

which is coupled with the equation for the electrostatic potential $\Delta \varphi=\operatorname{div}\left\{\operatorname{Im}\left[\psi^{*}(\nabla-\mathrm{iA}) \psi\right]\right\}$. The vector potential of the dipole is given by

$\mathbf{A}=\frac{m}{a} \frac{\mathbf{r} \times \hat{x}}{\rho^{2}}\left[\frac{x-a / 2}{\sqrt{\rho^{2}+(x-a / 2)^{2}}}-\frac{x+a / 2}{\sqrt{\rho^{2}+(x+a / 2)^{2}}}\right]$,

with $\rho^{2}=y^{2}+z^{2}$. Here, we express the length and time in units of the coherence length $\xi$ and the GL relaxation time $t_{G L}=\mu_{0} \lambda^{2} / \rho_{n}\left(\rho_{n}\right.$ is the normal-state resistivity), respectively. The electrostatic potential $\varphi$ and the vector potential $\mathbf{A}$ are scaled to $\varphi_{0}=\Phi_{0} / 2 \pi t_{G L}$ and $\Phi_{0} / 2 \pi \xi$, respectively. The order parameter is in units of $\psi_{0}$ which is the value of the order parameter at zero applied field and current. The coefficient $u$, which governs the relaxation of the order parameter (i.e., the ratio between relaxation times for the phase and the amplitude of $\psi$ ), is taken $u=12$ which is found for dirty gapless superconductors within the microscopic BCS theory. ${ }^{27}$ Using the normal state resistivity $\rho_{n}=18.7 \mu \Omega \mathrm{cm}$, zero temperature coherence length $\xi(0)=10 \mathrm{~nm}$, and penetration depth $\lambda(0)=200 \mathrm{~nm}$, which are typical for $\mathrm{Nb}$ thin films, ${ }^{28}$ one can obtain $t_{G L} \approx 6.72 \mathrm{ps}$ and $\varphi_{0} \approx 50 \mu \mathrm{V}$. The magnetic moment of the dipole is given in units of $m_{0}=\Phi_{0} \xi / 2 \pi$. For the investigated $\mathrm{Nb}$ superconductor $m_{0} \approx 3.3 \times 10^{-24} \mathrm{~A} \cdot \mathrm{m}^{2}$, and so, the field generated at the surface, $B_{S} \sim m_{0} / r^{3} \approx 3.3 \mathrm{~T}$ for $r \approx \xi(0)$. We solved the above equations self-consistently in a 3D cartesian grid (up to 129 grid points in each of the three directions) using semi-implicit Crank-Nicholson ${ }^{29}$ (for $\psi$ ) and multigrid (for $\varphi$ ) iterative methods. We use superconductingvacuum boundary condition $\left.(\nabla-i \mathbf{A}) \psi\right|_{n}=0$ and $\left.\nabla \varphi\right|_{n}=0$ at all sample boundaries [including boundaries of the cavity (see Fig. 4)], except at the current contacts where we use $\psi=0$ and $\left.\nabla \varphi\right|_{n}=-j$, with $j$ being the applied current density in units of $j_{0}=\Phi_{0} / 2 \pi \mu_{0} \xi \lambda^{2}$. The simulations are carried out in two steps, first we stabilize the vortex configuration for a given $m$ and next a current is applied, which is then increased from zero to its maximal value over the time interval $\Delta t=250 t_{G L}$.

A single dipole-vortex cutting and recombination. As a representative example, we consider a superconducting sample with dimensions $20 \xi \times 20 \xi \times 40 \xi$ and a magnetic dot of size $a=4 \xi$. We first discuss the results for the positive direction of the applied current $j^{+}$. Figure 2 shows the voltage vs time $V(t)$ characteristics of the sample for $j^{+}=0.2 j_{0}$ together with the evolution of the vortex state, through isosurface plots of the Cooper-pair density. The inhomogeneity of the magnetic field makes vortices enter the system in the form of closed loops (panel 1). The Lorentz force from the current acts in such a way that it expands the vortex loop (see the arrows in panel 1 for its direction) to the point of reaching the lateral surface (panel 2). Then it breaks into two vortex lines, connected to the dipole edges (see top vortices in panel 3), and a loosed vortex, completely detached from the dipole (bottom vortex in panel 3 ). This vortex cutting (due to the sample boundary) results in 


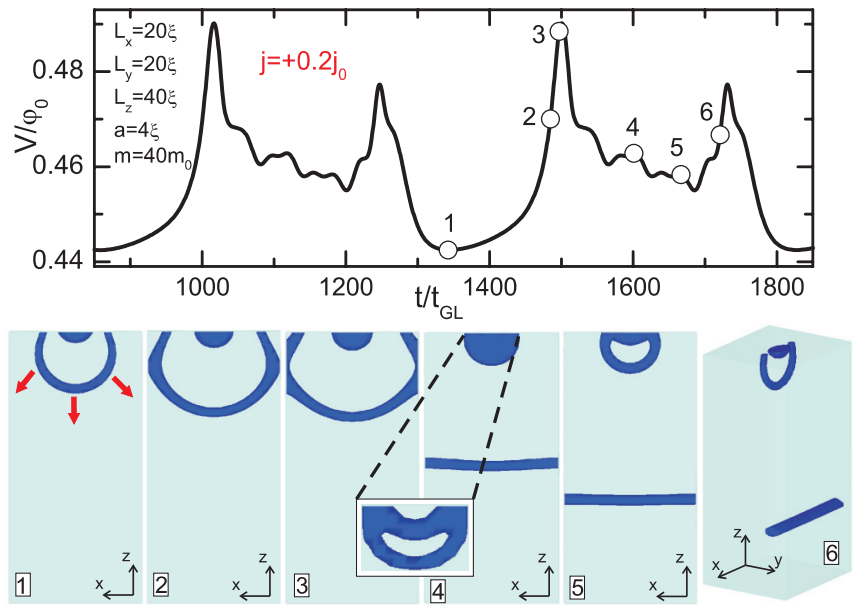

FIG. 2. (Color online) Voltage vs time characteristics of the sample (of size $L_{x}=20 \xi, L_{y}=20 \xi$, and $L_{z}=40 \xi$ ) with a magnetic dipole (of length $a=4 \xi$ ) on top for the positive current density $j=0.2 j_{0}$ and magnetic moment of the dipole $m=40 m_{0}$. Panels (1-6) show the isosurface plots of $|\psi|^{2}$ [light/dark blue (gray) corresponds to larger/smaller $|\psi|^{2}$ ] at the time intervals indicated in the main panel. Arrows in panel 1 show the direction of the Lorentz force acting on the vortex loops.

a pronounced peak in the $V(t)$ curve (point 3 ). The former connected two vortices move upwards in a contraction motion and the loosed vortex moves towards the bottom surface (panel 4). During the exit (panel 6) this vortex achieves its largest speed when there is no line tension to balance the Lorentz force, and this causes another peak in the voltage (point 6). After this voltage peak the system relaxes to its initial state, thus performing a periodic motion. One can also observe extra features in the $V(t)$ curve between the two peaks corresponding to vortex cutting and expulsion. These small oscillations are due to the formation of extra vortex loops while the loosed vortex moves along the sample (see panels 4 and 5). Initially, two vortex loops are formed near the dipole (panel 4), but one of them shrinks in favor of the growth of the other ${ }^{30}$ by the action of the positive current $j^{+}$. The amplitude of the voltage signal, as well as the period of the voltage oscillations, depends on the value of the relaxation parameter $u$ (see Eq. 1): The voltage signal increases and the period of the oscillations decreases with decreasing $u$.

For the negative direction of the applied current $j^{-}, V(t)$ exhibits periodic oscillations (one period is shown in Fig. 3). Now the Lorentz force works opposite and shrinks the existing loops inside the superconductor (see the arrows in panel 5), but at the same time, it is also able to cause the expansion of some entering vortex pairs (panel 1). By means of the Lorentz force this pair of vortices moves deeper inside the sample (panels 2 and 3), leading to an increased voltage signal [points 1-3 on the $V(t)$ curve], and eventually touch each other at some distance away from the dipole (panel 4). At this moment a vortex cutting and recombination process unfolds as the two upper parts of the two vortices recombine in a new loop that begins and ends in the dipole (panel 5). The two lower pieces of the vortex lines join into a loosed vortex loop, which moves towards the bottom of the sample due to the Lorentz force (see the arrows in panel 5), resulting in a peak in the output voltage. As in the case of positive current, the largest voltage
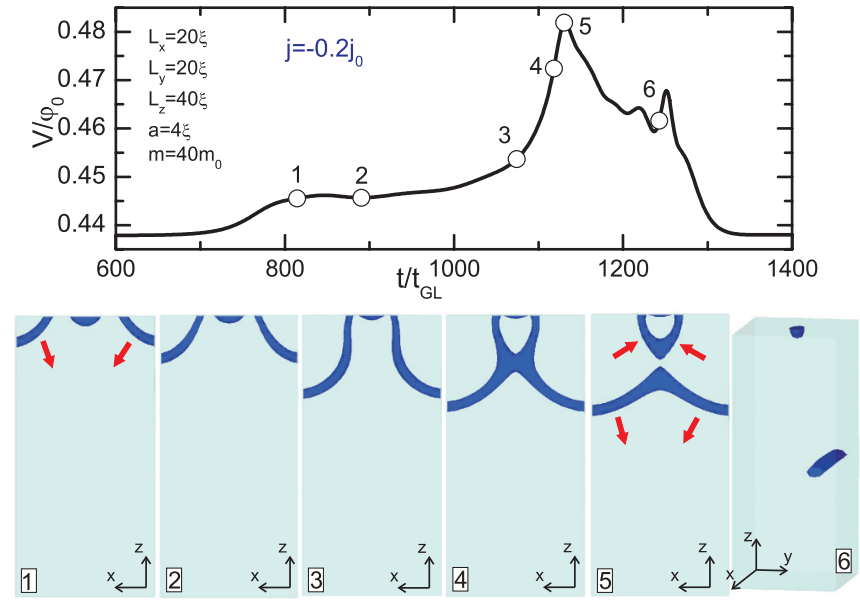

FIG. 3. (Color online) The same as in Fig. 2 but for negative direction of the applied current.

signal corresponds to the vortex cutting process (point 5). For the given parameters of the sample and the applied current the minimum of the voltage corresponds to the Meissner state. Note that the finite voltage in the latter state is due to the normal contacts (i.e., we performed two probe measurements) and that for both directions of the applied current the stripped vortex loop is not able to reach the bottom surface. Instead it leaves the sample through the side surface (panel 6 in Figs. 2 and 3 ), because near to the bottom the magnetic field of the dipole becomes weak and unable to sustain the vortex line. The time required for this vortex line to exit the sample is larger than the time for the inner loop to achieve full contraction.

The fast vortex dynamics can be slowed down by adding a pinning center in the lower part of the sample, taken in our simulations as a cavity of size $8 \xi \times 8 \xi \times 8 \xi$. Figure 4 shows the voltage characteristics of the sample together with the evolution of the vortex state for the positive direction of the current. The effect of the cavity is quite obvious from this figure: (i) Extra peaks appear in the voltage curve (points 4

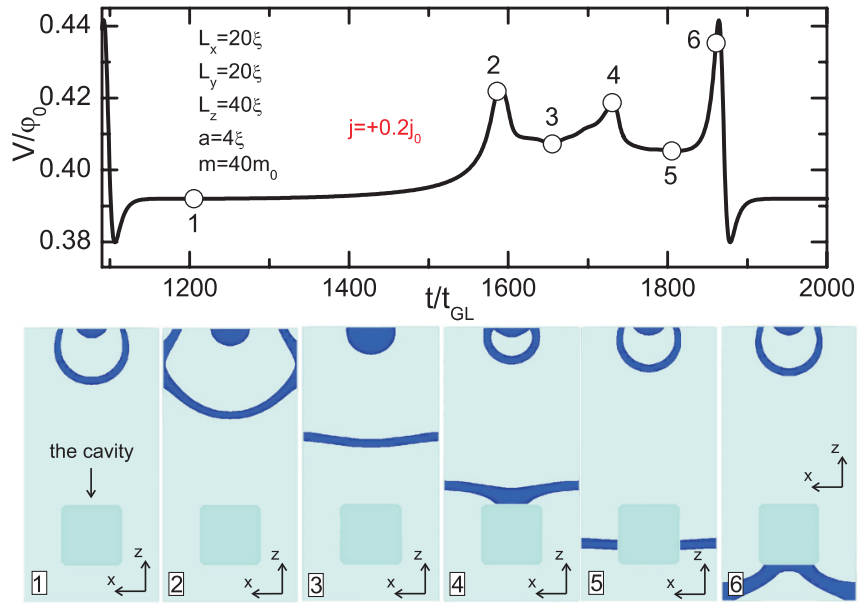

FIG. 4. (Color online) $V(t)$ curve of the sample (of size $20 \xi \times$ $20 \xi \times 40 \xi$ ) with a cavity (of size $8 \xi \times 8 \xi \times 8 \xi$ ) in the lower part of the sample for $m=40 m_{0}$ and $j=0.22 j_{0}$. Panels (1-6) show the isosurface plots of $|\psi|^{2}$ at the time intervals indicated in the main panel. 
and 6) corresponding to the pinning (panel 4) and depinning (panel 6) processes. Since the depinning process follows by the expulsion of the vortex from the sample (panel 6), the voltage signal is larger than the one corresponding to the vortex cutting (due to the sample edge) process (point 2). (ii) The period of the voltage oscillations $\Delta t$ increases due to the temporary pinning of the vortex by the cavity (panel 5) (in this particular case $\Delta t$ increased by $60 \%$ ). Permanent trapping of the loosed vortex is not possible here due to the larger applied current. The important point is that the detection of the loosed vortex through any of the available techniques, such as scanning tunneling microscopy, ${ }^{31}$ magnetic force microscope, ${ }^{32}$ or high spatial resolution scanning Hall probe microscopy, ${ }^{33}$ provides a clear signal of the 3D features of vortex lines. Interestingly, a setup, similar to the present one, consisting of a dirty niobium bridge with a magnetized cobalt strip on the top, has been proposed and shown to enhance critical current values as compared to the bulk. ${ }^{34}$

Multiple dipoles-extended vortex loops. Vortex-antivortex patterns can be created near periodic superconductor/ferromagnet hybrid structures, ${ }^{35,36}$ and here we find that the vortex-antivortex pair signals the presence of confined vortex loops, and their subsequent cutting and recombination process, here studied for a positive current $j^{+}$leading to a detectable voltage signal. As a representative example, we take a superconducting block (of size $64 \xi \times 16 \xi \times 16 \xi$ ) with three co-linear magnetic dipoles (of length $a=4 \xi$ ) (see panel 1 in Fig. 5). In Fig. 5 the time evolution of the output voltage for the dipoles, $m=40 m_{0}$, under the current density $j^{+}=0.22 j_{0}$, and snapshots of the Cooper-pair density at time intervals indicated in the $V(t)$ curve. As in the previous examples, vortices enter the system in the form of confined loops (panel 1), and expand because of the Lorentz force. When they touch each other (panel 2), the loops are cut and recombine into a single big vortex loop (panel 3) that expands (panel 4) and finally reaches the bottom surface (panel 5). At this moment two top to bottom lines are detached, and they correspond to a vortex-antivortex pair (panel 6). Their motion towards the edge and disappearance there (panels 7 and 8 ) corresponds to a nearly flat voltage plateau, as seen in the main panel. Instead a peak in the $V(t)$ curve is present from the moment the three loops touch each other to the release of the vortex-antivortex pair. The observed presence of a vortex line at the bottom surface, where before there was none, provides simple and straightforward proof of this cutting and recombination process.

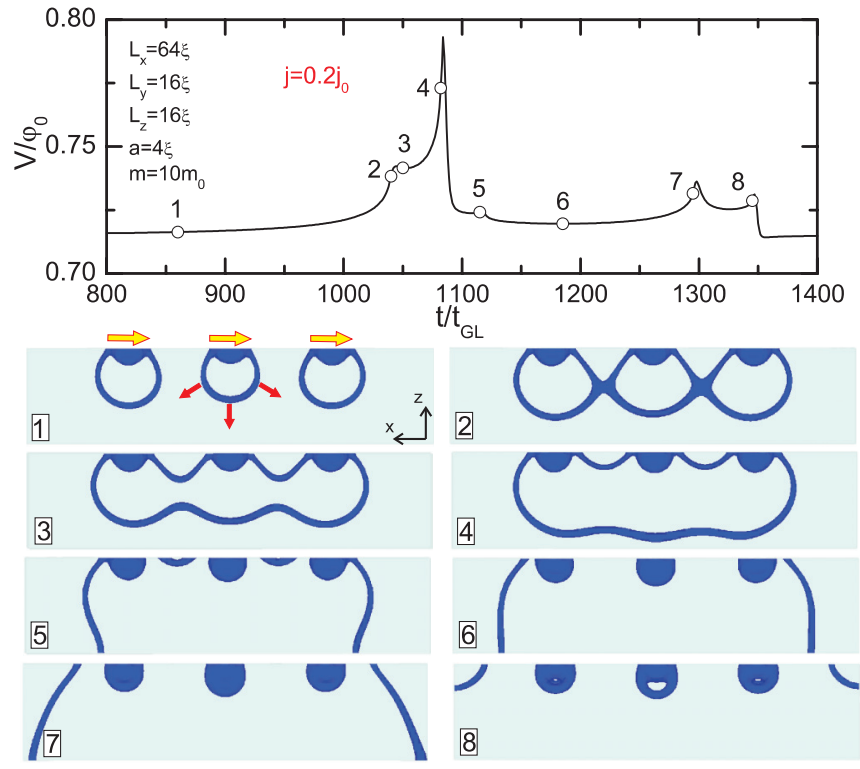

FIG. 5. (Color online) Evolution of vortex loops (panels 1-8) in the sample with dimensions $64 \xi \times 16 \xi \times 16 \xi$ with three magnetic dipoles of length $a=4 \xi$ and magnetization $m=40 m_{0}$ for $j=$ $0.22 j_{0}$. Arrows on top of panel 1 illustrate the location of the dipoles. Main panel shows the $V(t)$ curve of the sample.

Conclusion. We have studied cutting and recombination of vortex loops in a superconductor-ferromagnet heterostructure. The presence of an external current applied perpendicular to the magnetic dipole put on top of a mesoscopic superconductor unfolds the $3 \mathrm{D}$ time evolution of vortex loops that can be detected through the analysis of voltage vs time characteristics curves. Vortices appear at surfaces where they were not present before the application of the current and can be pinned there. Then well established methods of vortex visualization can be used to detect the pinned vortices that result from cutting and recombination processes. We expect that the present scenario of vortex cutting and recombination will be helpful to understand the spontaneous vortex phase ${ }^{37}$ of superconductors containing magnetic elements ${ }^{38,39}$ or artificially made magnetic inclusions. ${ }^{40}$

Acknowledgments. This work was supported by the Flemish Science Foundation (FWO-Vl) and the bilateral programme between Flanders and Brazil. G.R.B. acknowledges support from FWO-Vl. A.R. de C.R. acknowledges CNPq for financial support.

\footnotetext{
* Deceased.

${ }^{\dagger}$ francois.peeters@ua.ac.be

${ }^{1}$ E. D. Siggia, Phys. Fluids 28, 794 (1985).

${ }^{2}$ I. Giaever, Phys. Rev. Lett. 15, 825 (1965).

${ }^{3}$ R. Deltour and M. Tinkham, Phys. Rev. 174, 478 (1968).

${ }^{4}$ J. W. Ekin, B. Serin, and J. R. Clem, Phys. Rev. B 9, 912 (1974).

${ }^{5}$ S. Kida and M. Takaoka, Phys. Fluids 30, 2911 (1987).

${ }^{6}$ S. Kida, M. Takaoka, and F. Hussain, Phys. Fluids A 1, 630 (1989).

${ }^{7}$ W. T. Ashurst and D. I. Meiron, Phys. Rev. Lett. 58, 1632 (1987).

${ }^{8}$ K. W. Schwarz, Phys. Rev. B 38, 2398 (1988).
}

${ }^{9}$ D. Kivotides, Phys. Rev. Lett. 96, 175301 (2006).

${ }^{10}$ S. Z. Alamri, A. J. Youd, and C. F. Barenghi, Phys. Rev. Lett. 101, 215302 (2008).

${ }^{11}$ M. S. Paoletti, M. E. Fisher, K. R. Sreenivasan, and D. P. Lathrop, Phys. Rev. Lett. 101, 154501 (2008).

${ }^{12}$ M. Paoletti, M. E. Fisher, and D. Lathrop, Physica D 239, 1367 (2010).

${ }^{13}$ G. Blatter, M. V. Feigel'man, V. B. Geshkenbein, A. I. Larkin, and V. M. Vinokur, Rev. Mod. Phys. 66, 1125 (1994). 
${ }^{14}$ M. J. Van Bael, J. Bekaert, K. Temst, L. Van Look, V. V. Moshchalkov, Y. Bruynseraede, G. D. Howells, A. N. Grigorenko, S. J. Bending, and G. Borghs, Phys. Rev. Lett. 86, 155 (2001).

${ }^{15}$ J. R. Clem, M. Weigand, J. H. Durrell, and A. M. Campbell, Supercond. Sci. Technol. 24, 062002 (2011).

${ }^{16}$ A. M. Campbell, Supercond. Sci. Technol. 24, 091001 (2011).

${ }^{17}$ H. S. Ruiz and A. Badia-Majos, Supercond. Sci. Technol. 23, 105007 (2010).

${ }^{18}$ E. H. Brandt and G. P. Mikitik, Phys. Rev. B 76, 064526 (2007).

${ }^{19}$ A. V. Silhanek, V. N. Gladilin, J. Van De Vondel, B. Raes, G. W. Ataklti, W. Gillijns, J. Tempere, J. T. Devreese, and V. V. Moshchalkov, Supercond. Sci. Technol. 24, 024007 (2011). M. V. Milošević and F. M. Peeters, Physica C 437-438, 208 (2006); M. V. Milošević, G. R. Berdiyorov, and F. M. Peeters, Phys. Rev. Lett. 95, 147004 (2005).

${ }^{20}$ R. Cubitt, A. S. Campbell, E. M. Forgan, C. D. Dewhurst, and G. Yang, Supercond. Sci. Technol. 22, 034014 (2009).

${ }^{21}$ J. R. Clem, Phys. Rev. Lett. 38, 1425 (1977).

${ }^{22}$ E. H. Brandt, J. Low Temp. Phys. 39, 41 (1980).

${ }^{23}$ G. E. Marsh, Phys. Rev. B 50, 571 (1994).

${ }^{24}$ Y. A. Genenko, Phys. Rev. B 53, 9473 (1996).

${ }^{25}$ A. Palau, R. Dinner, J. H. Durrell, and M. G. Blamire, Phys. Rev. Lett. 101, 097002 (2008).

${ }^{26}$ L. Kramer and R. J. Watts-Tobin, Phys. Rev. Lett. 40, 1041 (1978); R. J. Watts-Tobin, Y. Krhenbhl, and L. Kramer, J. Low Temp. Phys. 42, 459 (1981).

${ }^{27}$ L. P. Gor'kov and G. M. Éliashberg, Soviet JETP 27, 328 (1968).

${ }^{28}$ A. I. Gubin, K. S. Il'in, S. A. Vitusevich, M. Siegel, and N. Klein, Phys. Rev. B 72, 064503 (2005).
${ }^{29}$ T. Winiecki and C. Adams, J. Comput. Phys. 179, 127 (2002).

${ }^{30}$ M. M. Doria, A. R. De C. Romaguera, M. V. Milosevic, and F. M. Peeters, Europhys. Lett. 79, 47006 (2007).

${ }^{31}$ A. Troyanovski, J. Aarts, and P. Kes, Nature (London) 399, 665 (1999).

${ }^{32}$ A. Moser, H. J. Hug, I. Parashikov, B. Stiefel, O. Fritz, H. Thomas, A. Baratoff, H.-J. Güntherodt, and P. Chaudhari, Phys. Rev. Lett. 74, 1847 (1995).

${ }^{33}$ A. Oral, S. Bending, R. Humphreys, and M. Henini, Supercond. Sci. Technol. 10, 17 (1997).

${ }^{34}$ D. Y. Vodolazov, B. A. Gribkov, S. A. Gusev, A. Y. Klimov, Y. N. Nozdrin, V. V. Rogov, and S. N. Vdovichev, Phys. Rev. B 72, 064509 (2005).

${ }^{35}$ M. Vélez, J. I. Martín, J. E. Villegas, A. Hoffmann, E. M. González, J. L. Vicent, and Ivan K. Schuller, J. Magn. Magn. Mater. 320, 2547 (2008); A. Yu. Aladyshkin, A. V. Silhanek, W. Gillijns, and V. V. Moshchalkov, Supercond. Sci. Technol. 22, 053001 (2009).

${ }^{36}$ M. V. Milošević and F. M. Peeters, Phys. Rev. Lett. 93, 267006 (2004); J. S. Neal, M. V. Milošević, S. J. Bending, A. Potenza, L. S. Emeterio, and C. H. Marrows, ibid. 99, 127001 (2007).

${ }^{37}$ T. K. Ng and C. M. Varma, Phys. Rev. Lett. 78, 330 (1997).

${ }^{38}$ D. E. Moncton, D. B. McWhan, P. H. Schmidt, G. Shirane, W. Thomlinson, M. B. Maple, H. B. MacKay, L. D. Woolf, Z. Fisk, and D. C. Johnston, Phys. Rev. Lett. 45, 2060 (1980).

${ }^{39}$ J. W. Lynn, G. Shirane, W. Thomlinson, and R. N. Shelton, Phys. Rev. Lett. 46, 368 (1981).

${ }^{40}$ A. Palau, H. Parvaneh, N. A. Stelmashenko, H. Wang, J. L. Macmanus-Driscoll, and M. G. Blamire, Phys. Rev. Lett. 98, 117003 (2007) 